\title{
Coronary subclavian steal syndrome following coronary by-pass surgery
}

\author{
BJ Kneale, AT Irvine, DJ Coltart
}

\begin{abstract}
Summary
The coronary steal syndrome is an uncommon but well recognised occurrence following coronary artery by-pass surgery using the internal mammary artery. We report a case of coronary steal successfully treated with percutaneous transluminal angioplasty of a subclavian stenosis.
\end{abstract}

Keywords: coronary subclavian steal, angioplasty, coronary artery by-pass surgery

As experience in the use of the internal mammary artery as a by-pass graft has accumulated, it has become established as the preferred conduit for myocardial revascularisation. The syndrome of coronary steal due to arterial disease proximal to the origin of the internal mammary artery is now well recognised. We report a case of coronary steal syndrome, its treatment with percutaneous transluminal angioplasty, and review the literature relating to this important problem.

\section{Case report}

A 59-year-old man presented with a threemonth history of increasingly severe angina. He was an ex-smoker with a past history of transitional cell bladder carcinoma treated with endoscopic resection. An exercise stress test demonstrated inferolateral ST depression of $3 \mathrm{~mm}$ at three minutes. Coronary angiography was performed which showed three-vessel coronary disease and good left ventricular function.

Coronary artery by-pass surgery was performed in September 1994, consisting of three saphenous vein grafts to the right coronary artery, left ventricular branch of the right coronary artery, and obtuse marginal branch of circumflex, and a left internal mammary artery (IMA) graft to the left anterior descending coronary artery (LAD). Recovery was uneventful.

At review in January 1995 he complained of

St Thomas's Hospital, London, SE1 7EH, UK Cardiac Department BJ Kneale

DJ Coltart

Radiology Department AT Irvine

Correspondence to Dr DJ Coltart

Accepted 15 September 1995 lessness. At coronary angiography no saphenous vein graft could be identified. There was marked retrograde filling of the IMA graft to its origin (figure 1A). An arch aortogram demonstrated an $80 \%$ stenosis in the proximal subclavian artery (figure $2 \mathrm{~A}$ ). A critical stenosis was noted in the LAD distal to the insertion of the IMA graft, not visualised at the preoperative assessment. continued exertional chest pain and breath-

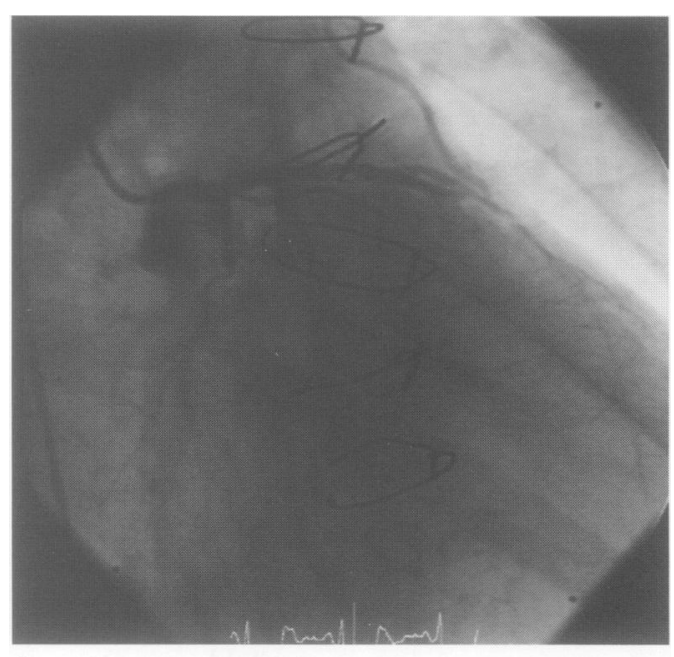

A

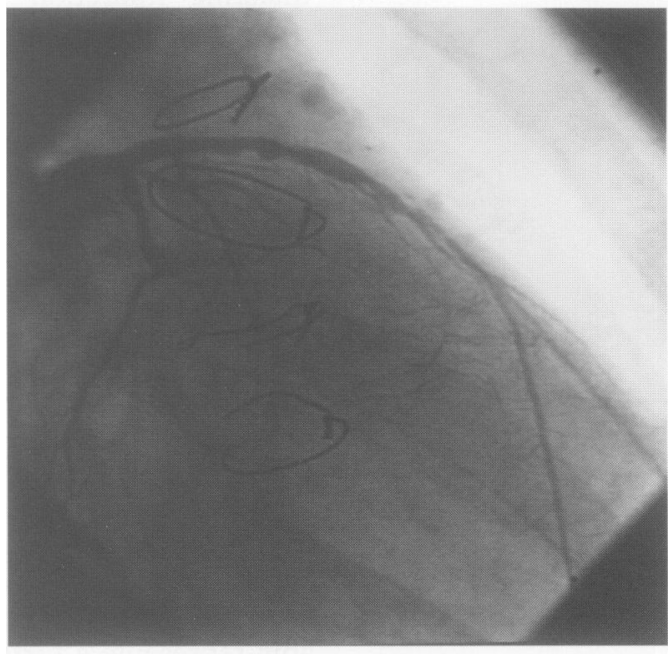

B

Figure 1 Coronary angiography: left coronary injection: (A) the IMA graft is demonstrated due to retrograde filling from the LAD but is not seen following angioplasty of the subclavian artery stenosis (B)

In view of these findings a combined approach was used to dilate both the left subclavian artery stenosis (figure $2 B$ ) and also the LAD stenosis distal to the insertion of the IMA graft. A right groin approach was used. The left subclavian stenosis was crossed with a hydrophilic guide wire and balloon dilatation were performed with a $6 \mathrm{~mm} \times 4 \mathrm{~cm}$ balloon, with a satisfactory result. On the initial flush series the left vertebral did not fill but showed normal filling after percutaneous transluminal angioplasty (PTA). Coronary angioplasty was 


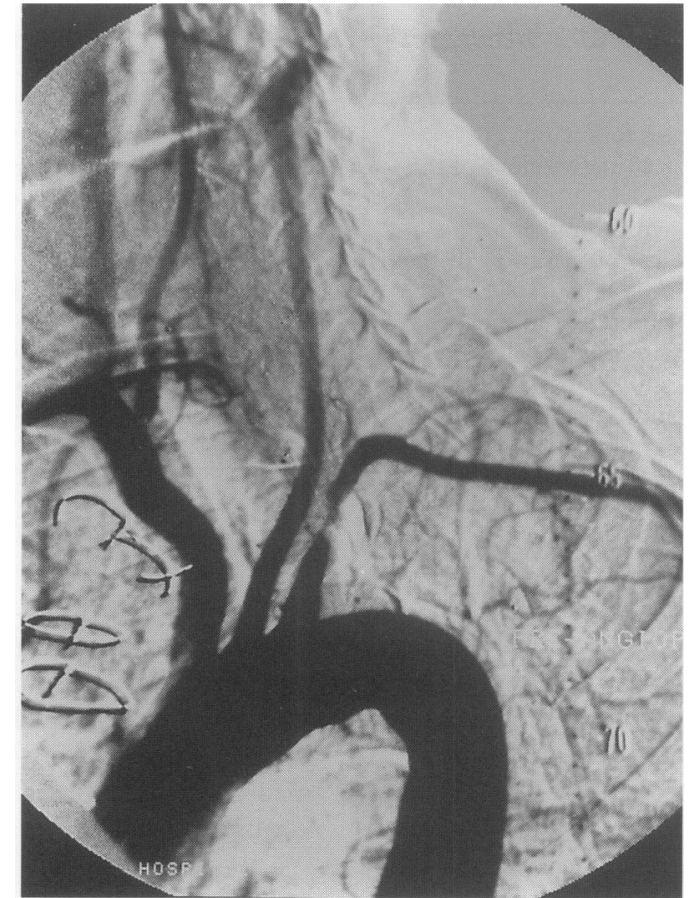

A

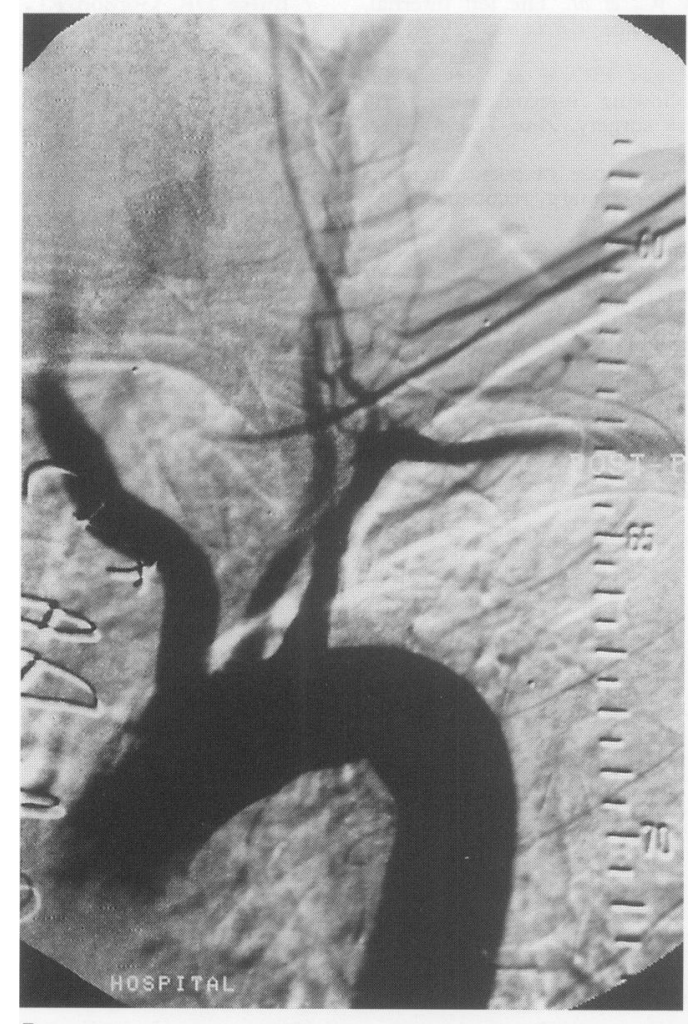

B

Figure 2 Digital subtraction angiography: flush aortogram demonstrating (A) an $80 \%$ left subclavian artery stenosis and absence of left vertebral artery filling treated with PTA with a good result and normal filling of the left vertebral (B).

subsequently performed to the distal LAD using a single inflation of a $2.5 \mathrm{~mm} \times 20 \mathrm{~mm}$ balloon. At this procedure no retrograde flow was seen in the IMA graft (figure 1B) and a good result from subclavian angioplasty was confirmed.

At follow-up the patient did not complain of exertional chest pain but his exercise tolerance remained limited by dyspnoea without evidence of cardiac failure. He did not have evidence of ischaemia on exercise testing and cardiac rehabilitation was recommended.

\section{Discussion}

The use of the internal mammary artery graft is widely accepted given its long-term patency rate and the low incidence of atherosclerosis in this vessel. ${ }^{1}$ Despite these advantages the efficacy of this vessel as a conduit can be drastically compromised by stenosis in the subclavian artery proximal to the origin of the IMA graft, resulting in the coronary steal syndrome. This syndrome is well described ${ }^{2}$ and it is estimated that approximately $0.5-$ $1.1 \%$ of patients referred for coronary artery by-pass surgery have a significant degree of subclavian artery stenosis. ${ }^{3}$

Initial reports on the treatment of coronary steal syndrome concentrated on the role of surgery with carotid - subclavian by-pass grafting as the preferred procedure. ${ }^{2,4}$ More recently the use of PTA has been advocated, the first report of successful treatment of coronary steal syndrome with PTA being published in $1986 .{ }^{5}$

A series of patients treated with subclavian artery PTA has been recently reported. ${ }^{6}$ In this study four patients had PTA to a single area of focal subclavian stenosis as treatment for coronary steal syndrome. In four other patients subclavian stenosis was treated before coronary artery by-pass surgery. All patients treated for coronary steal syndrome were free of myocardial ischaemia at follow-up (mean follow-up 39 months). Three of the four patients treated pre-operatively underwent successful coronary artery by-pass grafting with the ipsilateral IMA and were free of symptoms at follow-up (mean follow-up 14 months). There were no complications in this group. The authors suggest that the cerebral circulation may be protected from embolisation by a delay in the reversal of flow in cerebral vessels following PTA of the subclavian artery. Cerebral autoregulation is proposed as causing a gradual decrease in vascular resistance necessary for the development of antegrade flow. Others have reported a major complication rate of $<1 \%{ }^{7}$

Directional atherectomy has been used successfully to treat coronary steal syndrome ${ }^{8}$ and endoluminal stenting has been employed in conjunction with PTA and to treat restenosis at the site of previous PTA. ${ }^{9,10}$

With the widespread use of IMA grafting as the preferred conduit for myocardial revascularisation, efforts should focus on attempts at prevention of the potentially drastic complication of coronary steal syndrome. Routine auscultation for subclavian bruits and measurement of the blood pressure in both arms are deemed to be effective screening methods which should alert the physician or surgeon to the possibility of subclavian stenosis. ${ }^{2}$ This could prevent use of the ipsilateral IMA as a graft or indicate the need for prophylactic treatment of the stenosis by PTA, shown to be successful in a small number of patients. 


\section{Coronary subclavian steal syndrome: features}

- rapid recurrence of angina following coronary artery by-pass grafting with use of the IMA as a graft

- may be associated with symptoms of vertebrobasilar insufficiency on exertion of the left arm

- supraclavicular bruit or difference in blood pressures between the two arms usually present

- angiographically demonstrable subclavian artery stenosis with retrograde filling of the IMA graft on left coronary injection

In conclusion we believe that all patients considered for coronary artery by-pass surgery should have blood pressure measurements taken in both arms and auscultation for subclavian bruits as a routine. If this were undertaken before coronary angiography then arch aortography may provide additional valu-

1 Okies JE, Page US, Bigelow JC, et al. The left internal mammary artery: the graft of choice. Circulation 1984; 70 (suppl 1): 213 .

2 Olsen CO, Dunton RF, Maggs PR, et al. Review of coronary-subclavian steal following internal mammary coronary-subclavian steal following internal mammary artery coronary arte

3 Harjola PT, Valle M. The importance of aortic arch or subclavian angiography before coronary reconstruction. Chest 1974; 66: 436-8.

4 Granke K, Van Meter CH Jr, White CJ, et al. Myocardial ischaemia caused by post-operative malfunction of an internal mammary coronary artery graft. $f$ Vasc Surg 1990 11: $659-64$.

5 Meranze SG, McLean GK, Burke DR, Balloon dilatation of a subclavian artery stenosis proximal to an internal mammary - coronary artery by-pass graft. $\mathcal{F}$ Intervent Radiol 1986; 1: 83-4.

6 Hallisey MJ, Rees JH, Meranze SG, et al. Use of angioplasty in the prevention and treatment of coronary-subclavian steal syndrome. $₹$ Virol 1995; 6: $125-9$.

\section{Learning / summary points}

- the coronary-subclavian steal syndrome is an uncommon but important problem following coronary artery by-pass surgery

- percutaneous transluminal angioplasty of subclavian artery stenosis is a safe and effective treatment

- measuring the blood pressure in both arms and auscultation for subclavian bruits should be performed routinely before coronary artery by-pass grafting using the internal mammary artery

able information. PTA would seem to be a safe and efficacious treatment for coronary steal syndrome and may have a role in treatment prior to coronary by-pass surgery. The use of intra-arterial stenting may further improve the results of PTA.

7 Burke DR, Gordon RL, Mishkin JD, et al. Percutaneous transluminal angioplasty of subclavian arteries. Radiology 1987; 164: 699-704.

8 Breall JA, Grossman W, Stillman IE, et al. Atherectomy of the subclavian artery for patients with symptomatic coronary-subclavian steal syndrome. F Am Coll Cardiol 1993; 21: $1564-7$.

9 Kugelmass AD, Kim D, Kuntz RE, et al. Endoluminal stenting of a subclavian artery stenosis to treat ischaemia in the distribution of a patent left internal mammary graft. Cathet Cardiovasc Diagn 1994; 33: $175-7$.

10 Mufti SI, Young KR, Schulthesis T. Restenosis following subclavian artery angioplasty for treatment of coronarysubclavian steal syndrome: definitive treatment with $\mathrm{Pal}-$ maz-stent placement. Cathet Cardiovasc. Diagn 1994; 33: $172-4$. 